\title{
Numerical Methods for KdV Type Fractional Order Equation with a Nonlocal Viscous Term
}

\author{
Lin Fubiao \\ School of Mathematics and Statistical, Guizhou University of Finance and Econmics, Guiyang, \\ 550025, China. \\ E-mail: linfubiao0851@163.com
}

Keywords: Fractional equation; stability; spectral method; decay rates

\begin{abstract}
This paper is to study the numerical solution of the Fractional order equation with a nonlocal viscous term. It proposes a numerical scheme to solve this equation. A detailed analysis is carried out for this scheme, and it is proved that the scheme is unconditionally stable. The numerical results verify that the fractional order equation with a nonlocal viscous term is of order 1.5 , when a nonlocal viscous term does not exist, the scheme is of order 2. At last, the proposed methods are used to investigate the asymptotical decay rate of the solutions to fractional order equation with a nonlocal viscous term. This paper equally discusses the role of the diffusion terms, the geometric dispersion and the nonlinearity respectively. The performed numerical experiment confirms that the decay rates in $L^{2}$-norm, $L^{\infty}$-norm, and are very close to- 0.25 , and -0.5 respectively.
\end{abstract}

\section{Introduction}

The study of equations with nonlocal viscosity terms has always been a hot issue,one must consider the influence of dissipation mechanism when describing the propagation of small amplitude long waves in nonlinear dispersive media. This dissipation will affect the real solution, which will complicate the solution. Kakutani and Matsuuchi [1] first discussed the influence of viscosity on the water wave model, and proposed a viscous water wave model with diffusion and dispersion terms. Liu, or Filla [2], Saut, Bona et al [3] obtained a bounded water depth model with viscous terms, while Dutky and Dias [ 4 ] obtained a fluid boundary layer water wave model with viscous terms when studying water wave equations. Recently dutykh [5] and Chen et al. [6] obtained a kind of unidirectional wave equation from the free surface problem of a fluid boundary layer

$$
\partial_{t} u+\partial_{x} u+\beta \partial_{x}^{3} u+\frac{\sqrt{v}}{\sqrt{\pi}} \int_{0}^{t} \frac{\partial_{t} u}{\sqrt{t-s}} d s+u \partial_{x} u-\alpha \partial_{x}^{2} u=0
$$

Its main characteristic is the interaction between diffusion term and dispersion term. The physical explanation is that the viscous boundary layer has both diffusion and dispersion in the fluid. 
Dutykh[5],Chen[6] examined the adaptability of the equation and proved that the solution of the equation has the following properties

$$
t^{\frac{1}{2}}\|u(t)\|_{L_{x}^{\infty}}+t^{\frac{1}{4}}\|u(t)\|_{L_{x}^{2}} \leq C\left(u_{0}\right)
$$

Chen [7] proposed an explicit and implicit scheme of temporal difference and spatial spectrum method, but this scheme is conditionally stable and has no stability analysis at the same time. Goubet and Warnault [8] discuss a linear viscous asymptotic water wave model and propose an estimate of the decay rate of the solution. Dumont and Duval [9] introduce a scheme based on backward Euler method to discretize fractional order terms from a numerical point of view. The difficulty of the numerical scheme is that on the one hand, the constructed scheme must be as stable as possible, and on the other hand, the computational complexity is as small as possible. An unconditionally stable numerical scheme for a class of viscous BBM equations Zhang and $\mathrm{Xu}$ [10] is proposed. For the semi-stable treatment of nonlinear terms, Taylor expansion of integral remainder terms is used. On this basis, we are prepared to solve this class of KdV equations by the above method.

In this paper, an unconditionally stable numerical scheme for viscous $\mathrm{KdV}$ equations is proposed to study the decay rate of the equations. The advantage of the scheme is that we can not only select a larger time step, but also analyze the stability of the scheme and prove that the schemes are unconditionally stable. Numerical results show that the time scheme is 1.5 order when there are fractional terms in the equations, and the time scheme is 2 order when there are no fractional terms. Finally, we use numerical examples to discuss the long-time decay rate of the solutions of these two types of equations, and discuss the influence of different parameters on the decay rate of the solutions. Numerical results show the decay rate of the solutions of these models.

The structure of this paper is as follows: In the next section, we will give the KdV equation studied. In the third section, the time discrete scheme and stability estimation of KDV equation will be given. In the fourth section, we will give some numerical results. Finally, we give some conclusions.

\section{KdV Fractional Order Equation with viscous}

We consider the following viscous $\mathrm{KdV}$ - type fractional order equation:

$$
\partial_{t} u+\partial_{x} u+\beta \partial_{x}^{3} u+\frac{\sqrt{v}}{\sqrt{\pi}} \int_{0}^{t} \frac{\partial_{t} u}{\sqrt{t-s}} d s+u \partial_{x} u-\alpha \partial_{x}^{2} u=0, t \in(0, T], x \in \Lambda,
$$

Satisfy the following initial conditions:

$$
u(x, 0)=u_{0}(x), x \in \bar{\Lambda},
$$

And boundary conditions:

$$
u(x, t)=u(x+L, t), t \in(0, T], x \in \bar{\Lambda},
$$

Where $\Lambda=(0, L), \bar{\Lambda}=[0, L], \quad T$ represents time,$\frac{\sqrt{v}}{\sqrt{\pi}} \int_{0}^{t} \frac{\partial_{t} u}{\sqrt{t-s}} d s$ is a fractional derivative term. $\alpha, \beta$ are non-negative constants. 


\section{Time Unconditionally Stable Discrete Scheme}

For a given positive integer $\mathrm{M}, t_{n}=n \Delta t, n=0,1, \cdots, M$, where $\Delta t=T / M$ be time step, using Taylor expansion we have

$$
u(t)=u(s)+\partial_{s} u(s)(t-s)+\int_{s}^{t} \partial_{\tau}^{2} u(\tau)(t-\tau) d \tau, \forall t, s \in(0, T]
$$

Let $t=t_{j}, t=t_{j+1}$, we have

$$
\begin{aligned}
\partial_{s} u(x, s) & =\frac{u\left(x, t_{j+1}\right)-u\left(x, t_{j}\right)}{\Delta t}-\frac{1}{\Delta t} \int_{s}^{t_{j+1}} \partial_{\tau}^{2} u(x, \tau)\left(t_{j+1}-\tau\right) d \tau \\
& +\frac{1}{\Delta t} \int_{s}^{t_{j}} \partial_{\tau}^{2} u(x, \tau)\left(t_{j}-\tau\right) d \tau
\end{aligned}
$$

Lin et al [ 11 ] proposed the following fractional derivative approximation:

$$
\begin{aligned}
\frac{1}{\Gamma\left(\frac{1}{2}\right)} \int_{0}^{t_{n+1}} \frac{\partial_{s} u(s)}{\sqrt{t-s}} d s & =\frac{1}{\Gamma\left(\frac{1}{2}\right)} \sum_{j=0}^{n} \int_{t_{j}}^{t_{j+1}} \frac{\partial_{s} u(s)}{\sqrt{t_{n+1}-s}} d s \\
& =\frac{1}{\Gamma\left(\frac{1}{2}\right)} \sum_{j=0}^{n} \frac{u\left(x, t_{j+1}\right)-u\left(x, t_{j}\right)}{\Delta t} \int_{t_{j}}^{t_{j+1}} \frac{1}{\sqrt{t_{n+1}-s}} d s+r^{n+1} \\
& =\frac{1}{\Gamma\left(\frac{3}{2}\right)} \sum_{j=0}^{n} \frac{u\left(x, t_{n+1-j}\right)-u\left(x, t_{n-j}\right)}{\Delta t^{1 / 2}}\left[(j+1)^{1 / 2}-j^{1 / 2}\right]+r^{n+1} \\
& =\frac{1}{\Gamma\left(\frac{3}{2}\right)} \sum_{j=0}^{n} a_{j} \frac{u\left(x, t_{n+1-j}\right)-u\left(x, t_{n-j}\right)}{\Delta t^{1 / 2}}+r^{n+1} \\
& =\frac{\Delta t^{-1 / 2}}{\Gamma\left(\frac{3}{2}\right)}\left(u\left(x, t_{n+1}\right)-\sum_{j=0}^{n-1}\left(a_{j}-a_{j+1}\right) u\left(x, t_{n-j}\right)-a_{n} u\left(x, t_{0}\right)\right)+r^{n+1}
\end{aligned}
$$

Where

$$
\begin{aligned}
& a_{j}=(j+1)^{1 / 2}-j^{1 / 2}, \\
& r^{n+1}=\frac{1}{\Gamma\left(\frac{1}{2}\right) \Delta t} \sum_{j=0}^{n}\left[-\int_{t_{j}}^{t_{j+1}} \int_{s}^{t_{j+1}} \partial_{\tau} u(x, \tau) \frac{\left(t_{k+1}-\tau\right)}{\sqrt{t_{n+1-s}}} d \tau d_{s}+\int_{t_{j}}^{t_{j+1}} \int_{s}^{t_{j}} \partial_{\tau}^{2} u(x, \tau) \frac{\left(t_{j}-\tau\right)}{\sqrt{t_{n+1}-s}} d \tau d s\right]
\end{aligned}
$$

Here, a linearized time semi-discrete scheme of the equations is introduced and their stability is examined.

Crank-Nicolson Scheme: We consider the following semi-implicit scheme based on Crank Nicolson method: 


$$
\begin{aligned}
& \frac{u^{n+1}-u^{n}}{\Delta t}+\partial_{x} u^{n+\frac{1}{2}}+\beta \partial_{x}^{3} u^{n+\frac{1}{2}}+\frac{v^{1 / 2} \Delta t^{-1 / 2}}{\Gamma\left(\frac{3}{2}\right)}\left(u^{n+1 / 2}-\sum_{j=0}^{n-1}\left(a_{j}-a_{j+1}\right) u^{n-1 / 2-j}-a_{n} u^{0}\right) \\
& +\frac{1}{6}\left(2 \partial_{x} u^{n+\frac{1}{2}}\left(3 u^{n}-u^{n-1}\right)+u^{n+\frac{1}{2}} \partial_{x}\left(3 u^{n}-u^{n-1}\right)\right)-\alpha \partial_{x}^{2} u^{n+\frac{1}{2}}=0, \quad n=1,2, \cdots M-1,
\end{aligned}
$$

Where

$$
u^{n+\frac{1}{2}}=\frac{u^{n+1}+u^{n}}{2}
$$

For the first step

$$
\frac{u^{1}-u^{0}}{\Delta t}+\partial_{x} u^{1}+\beta \partial_{x}^{3} u^{1}+\frac{v^{1 / 2} \Delta t^{-1 / 2}}{\Gamma\left(\frac{3}{2}\right)}\left(u^{1}-a_{0} u^{0}\right)+\frac{1}{3}\left(2 u^{0} \partial_{x} u^{1}+u^{1} \partial_{x} u^{0}\right)-\alpha \partial_{x}^{2} u^{1}=0
$$

Theorem 3.1: Time semi-discrete schemes (4) - (5) is unconditionally stable, i.e.:

$$
\begin{gathered}
\left\|u^{1}\right\|_{0} \leq\left\|u^{0}\right\|_{0} \\
E\left(u^{n+1}\right) \leq E\left(u^{1}\right)+\frac{v^{\frac{1}{2}} T^{\frac{1}{2}}}{\Gamma\left(\frac{3}{2}\right)}\left\|u^{0}\right\|_{0}^{2}, \quad n=1,2, \cdots, M-1,
\end{gathered}
$$

Where

$$
\mu=\frac{v^{\frac{1}{2}} \Delta t^{\frac{1}{2}}}{\Gamma\left(\frac{3}{2}\right)}, E\left(u^{n}\right)=\left\|u^{n}\right\|_{0}^{2}+\mu \sum_{j=0}^{n-1} a_{j}\left\|u^{n-\frac{1}{2}-j}\right\|_{0}^{2}
$$

Proof: Taking the inner product with $2 \Delta t u^{1}$ in (5), noticing

$$
\begin{aligned}
& \left(2 \partial_{x} u^{1} u^{0}+u^{1} \partial_{x} u^{0}, u^{1}\right)=\left(\partial_{x} u^{1} u^{0}+\partial_{x}\left(u^{1} u^{0}\right), u^{1}\right)=\left(\partial_{x} u^{1} u^{0}, u^{1}\right)-\left(u^{1} u^{0}, \partial_{x} u^{1}\right)=0, \\
& \left(u^{1}-u^{0}, 2 u^{1}\right)=\left\|u^{1}\right\|_{0}^{2}-\left\|u^{0}\right\|_{0}^{2}+\left\|u^{1}-u^{0}\right\|_{0}^{2} .
\end{aligned}
$$

Then

$$
\left\|u^{1}\right\|_{0}^{2}-\left\|u^{0}\right\|_{0}^{2}+\left\|u^{1}-u^{0}\right\|_{0}^{2}+\mu\left(\left\|u^{1}\right\|_{0}^{2}-\left\|u^{0}\right\|_{0}^{2}+\left\|u^{1}-u^{0}\right\|_{0}^{2}\right)+2 \Delta t \alpha\left\|\partial_{x} u^{1}\right\|_{0}^{2}=0
$$

You can get (6) by removing some positive terms. Taking the inner product with $2 \Delta t u^{n+\frac{1}{2}}$ in (4), we have

$$
\begin{aligned}
& \left(2 \partial_{x} u^{n+\frac{1}{2}}\left(3 u^{n}-u^{n-1}\right)+u^{n+\frac{1}{2}} \partial_{x}\left(3 u^{n}-u^{n-1}\right), u^{n+\frac{1}{2}}\right) \\
& =\left(\partial_{x} u^{n+\frac{1}{2}}\left(3 u^{n}-u^{n-1}\right), u^{n+\frac{1}{2}}\right)+\left(\partial_{x}\left(u^{n+\frac{1}{2}}\left(3 u^{n}-u^{n-1}\right)\right), u^{n+\frac{1}{2}}\right) \\
& \left.=\left(\partial_{x} u^{n+\frac{1}{2}}\left(3 u^{n}-u^{n-1}\right), u^{n+\frac{1}{2}}\right)-\left(\left(3 u^{n}-u^{n-1}\right) u^{n+\frac{1}{2}}, \partial_{x} u^{n+\frac{1}{2}}\right)\right)=0 .
\end{aligned}
$$

That is 


$$
\left\|u^{n+1}\right\|_{0}^{2}-\left\|u^{n}\right\|_{0}^{2}+2 \mu\left(u^{n+1 / 2}-\sum_{j=0}^{n-1}\left(a_{j}-a_{j+1}\right) u^{n-\frac{1}{2}-j}-a_{n} u^{0}, u^{n+\frac{1}{2}}\right)+2 \Delta t \alpha\left\|\partial_{x} u^{n+\frac{1}{2}}\right\|_{0}^{2}=0 .
$$

Using Cauchy -Schwarz inequality, we have

$$
\begin{aligned}
\left\|u^{n+1}\right\|_{0}^{2}-\left\|u^{n}\right\|_{0}^{2}+2 \mu\left\|u^{n+\frac{1}{2}}\right\|_{0}^{2} & \leq \mu\left(\sum_{j=0}^{n-1}\left(a_{j}-a_{j+1}\right)\left(\left\|u^{n-\frac{1}{2}-j}\right\|_{0}^{2}+\left\|u^{n+\frac{1}{2}}\right\|_{0}^{2}+a_{n}\left(\left\|u^{0}\right\|_{0}^{2}+\left\|u^{n+\frac{1}{2}}\right\|_{0}^{2}\right)\right)\right) \\
& =\mu \sum_{j=0}^{n-1}\left(a_{j}-a_{j+1}\right)\left\|u^{n-\frac{1}{2}-j}\right\|_{0}^{2}+\mu\left(\left(1-a_{n}\right)+a_{n}\right)\left\|u^{n+\frac{1}{2}}\right\|_{0}^{2}+\mu a_{n}\left\|u^{0}\right\|_{0}^{2}
\end{aligned}
$$

That is

$$
\begin{array}{r}
\left\|u^{n+1}\right\|_{0}^{2}+\mu \sum_{j=0}^{n-1} a_{j+1}\left\|u^{n-\frac{1}{2}-j}\right\|_{0}^{2}+\mu\left\|u^{n+\frac{1}{2}}\right\|_{0}^{2} \leq\left\|u^{n}\right\|_{0}^{2}+\mu \sum_{j=0}^{n-1} a_{j}\left\|u^{n-\frac{1}{2}-j}\right\|_{0}^{2}+\mu a_{n}\left\|u^{n+\frac{1}{2}}\right\|_{0}^{2} \\
\text { Observe } \mu \sum_{j=0}^{n-1} a_{j+1}\left\|u^{n-\frac{1}{2}-j}\right\|_{0}^{2}+\mu\left\|u^{n+\frac{1}{2}}\right\|_{0}^{2}=\mu \sum_{j=1}^{n} a_{j}\left\|u^{n+\frac{1}{2}-j}\right\|_{0}^{2}+\mu\left\|u^{n+\frac{1}{2}}\right\|_{0}^{2}=\mu \sum_{j=0}^{n} a_{j}\left\|u^{n+\frac{1}{2}-j}\right\|_{0}^{2}
\end{array}
$$

Therefore, we have

$$
\left\|u^{n+1}\right\|_{0}^{2}+\mu \sum_{j=0}^{n} a_{j}\left\|u^{n+\frac{1}{2}-j}\right\|_{0}^{2} \leq\left\|u^{n}\right\|_{0}^{2}+\mu \sum_{j=0}^{n-1} a_{j}\left\|u^{n-\frac{1}{2}-j}\right\|_{0}^{2}+\mu a_{n}\left\|u^{0}\right\|_{0}^{2}
$$

The result of the theorem is obtained.

\section{Numerical results}

\subsection{Validity of numerical solution}

Here we discuss the spatial spectral discretization of numerical schemes (4) - (5). We use Fourier- Galerkin spectral method to discretize the spatial.

Let

$$
S_{N}=\operatorname{span}\{\exp (-i 2 \pi k x / L):-N / 2 \leq k \leq N / 2-1\} .
$$

C-N/F-G scheme: The Fourier spectral method of scheme (4) is:Find $u_{N}^{0}=\pi_{N} u_{0}$, such that $u_{N}^{n+1} \in S_{N}$,

$$
\begin{aligned}
& \left(\frac{u_{N}^{n+1}-u_{N}^{n}}{\Delta t}, \psi_{N}\right)+\left(\partial_{x} u_{N}^{n+\frac{1}{2}}, \psi_{N}\right)+\beta\left(\partial_{x}^{3} u_{N}^{n+\frac{1}{2}},, \psi_{N}\right)+\frac{v^{1 / 2} \Delta t^{-1 / 2}}{\Gamma\left(\frac{3}{2}\right)}\left(u_{N}^{n+\frac{1}{2}}-\sum_{j=0}^{n-1}\left(a_{j}-a_{j+1}\right) u_{N}^{n-\frac{1}{2}-j}-a_{n} u_{N}^{0}, \psi_{N}\right) \\
& +\frac{1}{6}\left(2 \partial_{\chi} u_{N}^{n+\frac{1}{2}}\left(3 u_{N}^{n}-u_{N}^{n}\right)+u_{N}^{n+\frac{1}{2}} \partial_{x}\left(3 u_{N}^{n}-u_{N}^{n-1}\right), \psi_{N}\right)+\alpha\left(\partial_{x}^{2} u^{n+\frac{1}{2}}, \psi_{N}\right)=0, n \geq 1, \psi_{N} \in S_{N} .
\end{aligned}
$$

Where $u_{N}^{n}(x)=\sum_{k=-N / 2}^{N / 2-1} \hat{u}_{k}^{n} \exp (-i 2 \pi k x / L)$, then we obtain $\left\{\hat{u}_{k}^{n+1}\right\}$ 


$$
\begin{aligned}
& \frac{1}{\Delta t}\left(\hat{u}_{k}^{n+1}-\hat{u}_{k}^{n}\right)+\left(i 2 \pi k / L+\alpha(2 \pi k / L)^{2}+\beta(2 \pi k / L)^{3}\right) \hat{u}_{k}^{n+\frac{1}{2}} \\
& +\frac{v^{1 / 2} \Delta t^{-1 / 2}}{\Gamma\left(\frac{3}{2}\right)}\left(\hat{u}_{k}^{n+\frac{1}{2}}-\sum_{j=0}^{n-1}\left(a_{j}-a_{j+1}\right) \hat{u}_{k}^{n-\frac{1}{2}-j}-a_{n} \hat{u}_{k}^{0}\right) \\
& +\frac{1}{6}\left\{2\left(3 u_{N}^{n}-u_{N}^{n-1}\right) \partial_{x} u_{N}^{n+\frac{1}{2}}+u_{N}^{n+\frac{1}{2}} \partial_{x}\left(3 u_{N}^{n}-u_{N}^{n-1}\right)\right\}_{k}=0,
\end{aligned}
$$

Where $\widehat{f}_{k}$ or $\{f\}_{k}$ represent the Fourier coefficients of $f$.

Because the exact solution of this kind of equation is difficult to find, we use the approximate method to calculate the convergence order of the numerical solution. Let

$$
\text { Rate }=\log _{2}\left(\frac{\left\|u_{N}^{n, 2 \Delta t}-u_{N}^{2 n, \Delta t}\right\|_{0}}{\left\|u_{N}^{2 n, \Delta t}-u_{N}^{4 n, \Delta t / 2}\right\|_{0}}\right),
$$

Table 1 C-N/F-G scheme The variation of convergence order of the scheme solution with time step

\begin{tabular}{|c|c|c|c|c|c|}
\hline$v \backslash \Delta t$ & $\Delta t=0.1$ & $\Delta t=0.05$ & $\Delta t=0.01$ & $\Delta t=0.005$ & $\Delta t=0.001$ \\
\hline$v=0$ & 2.0070 & 2.0045 & 2.0011 & 2.0005 & 2.0001 \\
\hline$v=1$ & 1.3638 & 1.3948 & 1.4466 & 1.4611 & 1.4818 \\
\hline
\end{tabular}

Let $u(x, 0)=0.32 \operatorname{sech}^{2}\left(0.4\left(x-x_{0}\right)\right)^{2}, x_{0}=L / 2, L=400, N=100, \alpha=\beta=1$.

It can be seen from the table that when the equation does not have fractional order terms, the time convergence order of the scheme is order 2 . When the equation contains fractional order terms, the time convergence order of the scheme is 1.5 order, The data in the table can prove that our scheme is valid.

\subsection{Decay rate of the solution}

We use numerical methods to verify the effectiveness of the method. First, we assume that

$$
\|u(\cdot, t)\|_{X}=C t^{\alpha}
$$

Calculate the following two norms

$$
R_{2}=\frac{\log \left(\frac{\|u(\cdot, t)\|_{0}}{\|u(\cdot, t-\Delta t)\|_{0}}\right)}{\log \left(\frac{t}{t-\Delta t}\right)}, R_{\infty}=\frac{\log \left(\frac{\|u(\cdot, t)\|_{L^{\infty}}}{\|u(\cdot, t)\|_{L^{\infty}}}\right)}{\log \left(\frac{t}{t-\Delta t}\right)},
$$

we can obtain the decay rate of the $L^{2}, L^{\infty}$. Let $u(x, 0)=0.32 \sec ^{2}\left(0.4\left(x-x_{0}\right)\right)^{2}$, $L=400, N=200, T=2000, \Delta t=5$. As shown in Table 4.1, we can see that $R_{2}, R_{\infty}$ approaches $-\frac{1}{4},-\frac{1}{2}$ respectively. 
Table 2 . The decay rate for various parameters

\begin{tabular}{|l|l|l|l|l|}
\hline$v$ & $\alpha$ & $\beta$ & $R_{2}$ & $R_{\infty}$ \\
\hline 1 & 0 & 0 & -0.2469 & -0.4909 \\
\hline 1 & 1 & 1 & -0.2431 & -0.4894 \\
\hline 1 & 0 & 1 & -0.2482 & -0.4961 \\
\hline 0.5 & 0.1 & 0.1 & -0.2510 & -0.4952 \\
\hline 0.5 & 1 & 0.1 & -0.2476 & -0.4941 \\
\hline 0.5 & 0.1 & 1 & -0.2523 & -0.4969 \\
\hline
\end{tabular}

4.3 Influence of parameters on decay rate

We will give various parameters $\alpha, \beta, v$ and let $T=2000$, $L=2000, N=200, \Delta t=5, u(x, 0)=0.32 \sec ^{2}\left(0.4\left(x-x_{0}\right)\right)^{2}$.
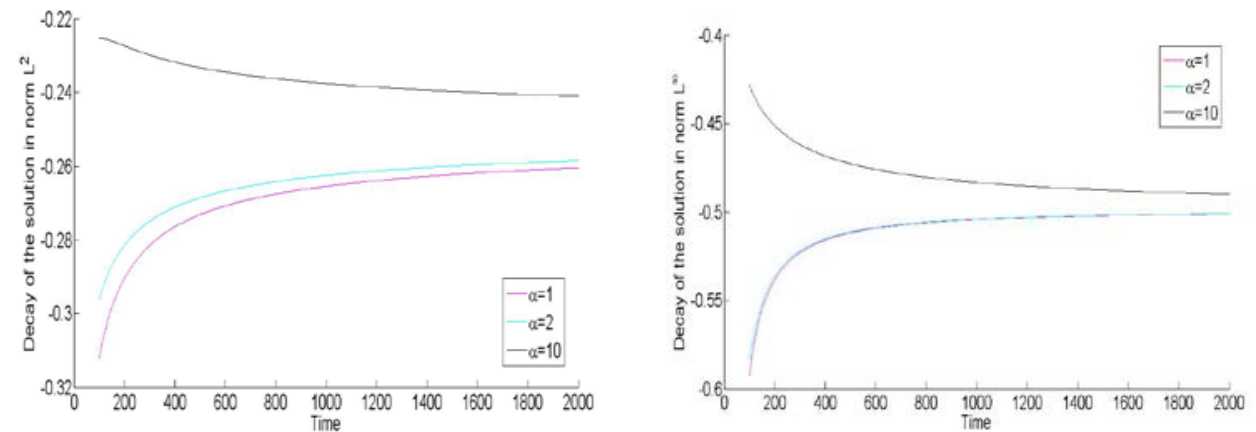

Fig 1. $\beta=v=1, R_{2}$ 与 $R_{\infty}$.
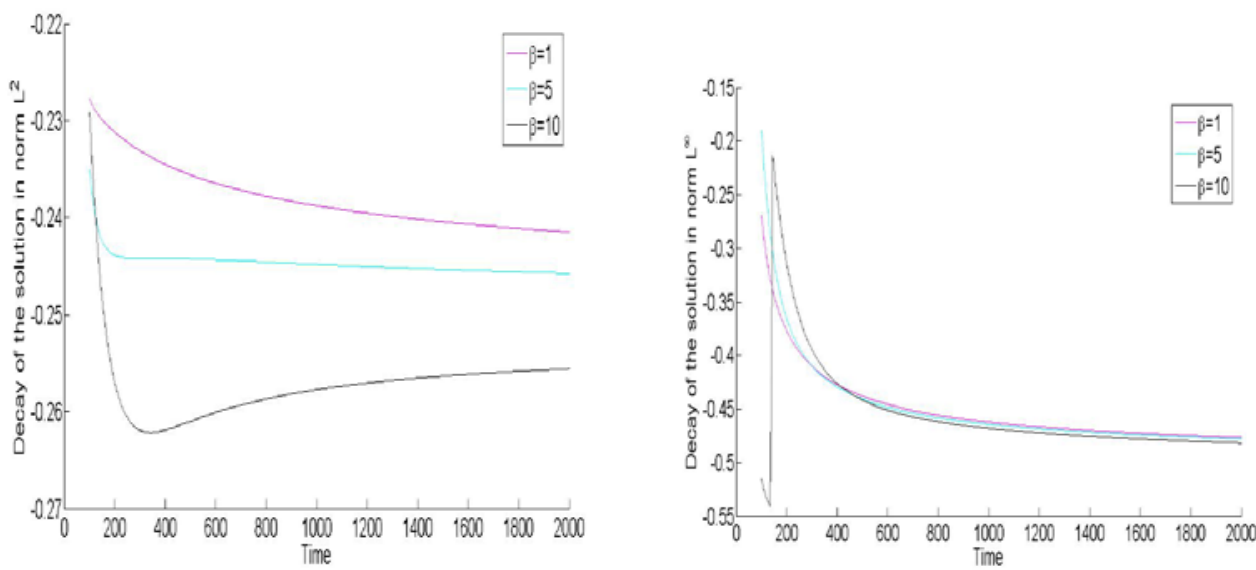

Fig 2. $\alpha=v=1, R_{2}$ 与 $R_{\infty}$. 

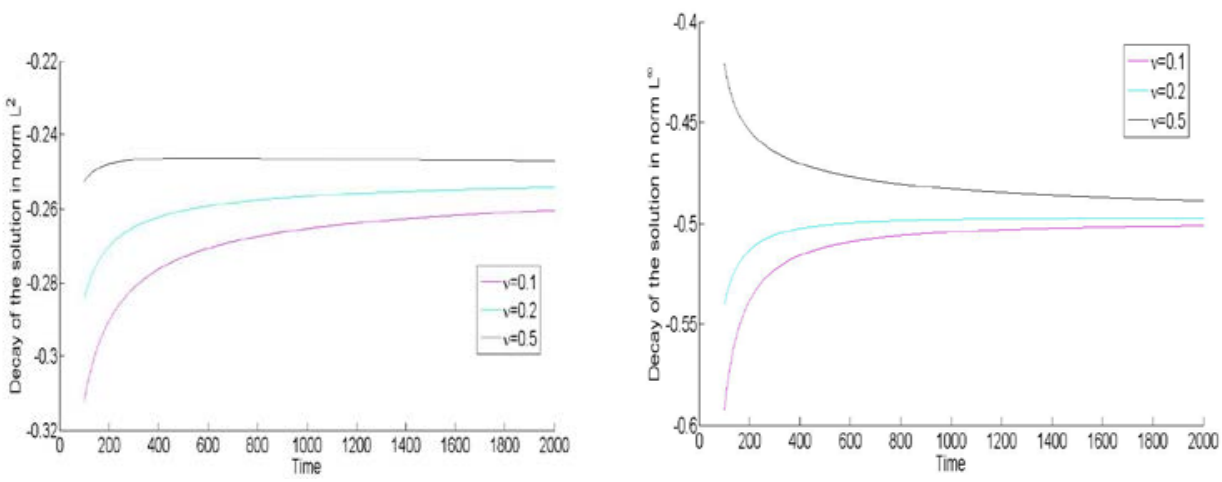

Fig 3. $\alpha=\beta=1, R_{2}$ 与 $R_{\infty}$..

As can be seen from fig 1-3, the decay rate of solutions are different, but they all fluctuate around -0.25 and -0.5 .

\section{Conclusion}

This paper presents an unconditionally stable numerical scheme for solving viscous KdV - type equations. The advantage of the scheme is that only one linear equation needs to be solved per iteration, which is especially suitable for solving viscous water wave equations in large time direction. Moreover, our numerical results verify the accuracy of the scheme. When there is a fractional term in the equation, the scheme is of order 1.5 in the time direction, and when there is no fractional term, the scheme is of order 2 in the time direction. Finally, we use the proposed numerical scheme to study the asymptotic decay rate of the solution. The numerical results are consistent with Chen [6] and others' theoretical proof.

\section{References}

[1] Kakutani T,Matsuuchi K.Efect of viscosity on long gravity waves[J].Journal of the Physical Society of Japan,1975,39(1):237-246.

[2] Liu $P$ L F,Orfila A.Viscous effects on transient long-wave propagation[J].Journal of Fluid Mechanics,2004,520(1):83-92.

[3] Saut J C,Bona J L,Chen M.Boussinesq equations and other systems for small-amplitude long waves in nonlinear dispersive media.I:derivation and linear theory[J].Journal of Nonlinear Science,2002,12(4):283-318

[4] Dutykh D,Dias F.Viscous potential free-surface flows in fluid layer of finite depth[J].Comptes Rendus Mathematique,2007,345(2):113-118.

[5] Dutykh D.Visco-potential free-surface flows and long wave modelling [J].European Journal of Mechanics B/Fluids,2009,28(3):430-443.

[6] Chen M,Dumont S,Dupaigne L,et 41.Decay of solutions to a water wave model with a nonlocal viscous dispersive term[J].Discrete and Continuous Dynamical Systems,2010,27(4):1473-1492.

[7] Chen M.Numerical investigation of a two-dimensional Boussinesq system [J].Discrete and Continuous Dynamical Systems,2009,28(4):1169-1190.

[8] Goubet O,Warnault G.Decay of solutions to a linear viscous asymptotic model for water waves[J].Chinese Annals of Mathematics-Series B,2010,31(6):841-85.

[9] Dumont S,Duval J B.Numerical investigation of the decay rate of solutions to models for water waves with nonlocal viscosity[J].International Journal of Numerical Analysis and Modeling,2013,1O(2):333-349.

[10] Zhang J, Xu C. Finite difference/spectral approximations to a water wave model with a nonlocal viscous term[J]. Applied Mathematical Modelling, 2014, 38(19): 4912-4925.

[11] Lin Y M,Xu C J.Finite difference/spectral approximations for the time-fractional diffusion equation[J].Journal of Computational Physics,2007,225(2):1533-1552. 